\title{
Neuroprotective Effect of the Endogenous Amine 1MeTIQ in an Animal Model of Parkinson's Disease
}

\author{
Agnieszka Wąsik $^{1}$ - Irena Romańska ${ }^{1} \cdot$ Jerzy Michaluk $^{1}$ - Agnieszka Zelek-Molik ${ }^{2}$. \\ Irena Nalepa ${ }^{2} \cdot$ Lucyna Antkiewicz-Michaluk $^{1}$
}

Received: 29 May 2015/Revised: 31 July 2015/ Accepted: 12 August 2015/Published online: 25 August 2015

(C) The Author(s) 2015. This article is published with open access at Springerlink.com

\begin{abstract}
Parkinson's disease (PD) is a neurodegenerative disorder that is hallmarked by pathological changes associated with the death of dopaminergic neurons, particularly in the extrapyramidal system (substantia nigra pars compacta, striatum) of the brain. Although the causes of slow neuronal death in PD are unknown, both genetic and environmental factors are likely involved. Endogenous isoquinolines, such as 1-benzyl-1,2,3,4-tetrahydroisoquinoline (1BnTIQ), present in the human brain have been previously reported to participate in the pathogenesis of PD. The chronic administration of 1BnTIQ induced parkinsonism in primates, and this effect might be associated with idiopathic PD. However, another endogenous derivative of tetrahydroisoquinoline, 1-methyl-1,2,3,4-tetrahydroisoquinoline (1MeTIQ), displays clear neuroprotective properties in the brain. In the present study, we investigated the neuroprotective effects of 1MeTIQ (25 and $50 \mathrm{mg} / \mathrm{kg}$ ) in an animal model of PD after the chronic administration of 1BnTIQ $(25 \mathrm{mg} / \mathrm{kg})$. Behavioral analyses demonstrate that both acute and repeated treatment with 1MeTIQ completely antagonized 1BnTIQ-induced changes in rat locomotor activity. Neurochemical experiments indicate that 1MeTIQ co-administered with 1BnTIQ completely antagonized 1BnTIQ-induced reduction in the dopamine (DA) concentration in rat brain structures. In
\end{abstract}

Agnieszka Wąsik

wasik@if-pan.krakow.pl

1 Department of Neurochemistry, Institute of Pharmacology Polish Academy of Sciences, 12 Smetna Street, 31-343 Krakow, Poland

2 Department of Biochemistry, Institute of Pharmacology Polish Academy of Sciences, 12 Smetna Street, 31-343 Krakow, Poland conclusion, the results demonstrate that 1MeTIQ possesses important neuroprotective properties in the animal model of PD and that the rats did not develop tolerance after its chronic administration.

Keywords 1-methyl-12,3,4-tetrahydroisoquinoline . 1-benzyl-1,2,3,4-tetrahydroisoquinoline · Parkinson's disease $\cdot$ Dopamine metabolism

\section{Introduction}

Tetrahydroisoquinolines, e.g., salsolinol and 1-benzyl1,2,3,4-tetrahydroisoquinoline (1BnTIQ), are a group of endogenous compounds with neurotoxic properties. However, one derivative from this group, 1-methyl-1,2,3,4-tetrahydroisoquinoline (1MeTIQ), exhibits neuroprotective properties. It has previously been shown that 1 MeTIQ acts as a reversible inhibitor of monoamino oxidase (MAO) activity, blocks both the formation of 3,4-dihydroxyphenylacetic acid (DOPAC) and the production of free radicals and shifts dopamine (DA) catabolism toward COMT-dependent $O$-methylation. This mechanism might be important for the neuroprotective activity of 1MeTIQ (Antkiewicz-Michaluk et al. 2001; Patsenka and Antkiewicz-Michaluk 2004). Additionally, 1MeTIQ acts as a natural scavenger of free radicals and interacts with the agonistic conformation of dopamine receptors (Antkiewicz-Michaluk et al. 2006, 2007). However, another endogenous amine present in the brain, 1BnTIQ, exhibits a contrasting molecular mechanism because this molecule enhances the rate of dopamine metabolism, activates the dopamine oxidation pathway, and increases the production of free radicals. In addition, 1BnTIQ inhibits the COMTdependent $O$-methylation pathway (Antkiewicz-Michaluk 
et al. 2001; Wąsik et al. 2009). Reflecting the neurotoxic mechanism of 1 BnTIQ, this compound is considered an etiological factor of idiopathic Parkinson's disease (PD) (Kotake et al. 1995). Previous studies have demonstrated that chronic treatment with 1BnTIQ produces parkinsonian-like symptoms in rodents and primates (Kotake et al. 1995; 2003; Kohta et al. 2010). In vitro studies indicated that 1BnTIQ induces cell death via apoptosis and produces an increase in the formation of the active caspase-3 protein fragments (Shavali and Ebadi 2003). 1BnTIQ accumulates in the dopaminergic neurons, where this molecule might exert pathological effects that lead to parkinsonism. Kotake et al. (1995) showed that the level of 1BnTIQ in the cerebrospinal fluid (CSF) of parkinsonian patients was three times higher than that in the CSF of the control group. These data suggest that chronic treatment with a low dose of 1BnTIQ might serve as an adequate animal model of the progressive process of $\mathrm{PD}$.

The aim of the present study was to investigate the neuroprotective effects of 1MeTIQ in an animal model of PD after the chronic administration of 1BnTIQ. Using behavioral tests, we analyzed the influence of acute and chronic treatment with 1MeTIQ on changes in rat locomotor activity induced through the repeated administration of 1BnTIQ.

In neurochemical experiments, using high-performance liquid chromatography (HPLC) methodology with electrochemical detection, we investigated the effect of 1MeTIQ on disturbances in brain DA metabolism evoked through 1BnTIQ. Additionally, the concentration of excitatory amino acids (EAA) in the frontal cortex and the level of $\alpha$-synuclein and tyrosine hydroxylase (TH) in the substantia nigra were also detected.

\section{Materials and Methods}

\section{Animals and Treatments}

All experiments were performed in male Wistar rats with an initial body weight of 220-240 g. All animals had free access to standard laboratory food and tap water and were kept at room temperature $\left(22^{\circ} \mathrm{C}\right)$ under an artificial light/dark cycle $(12 / 12 \mathrm{~h}$, light on at 7:00).

1BnTIQ was intraperitoneally (i.p.) administered at a dose of $25 \mathrm{mg} / \mathrm{kg}$ chronically for 14 consecutive days. In the mixed group, a single or chronic dose of 1MeTIQ (25 or $50 \mathrm{mg} / \mathrm{kg}$ i.p.) was administered $20 \mathrm{~min}$ before $1 \mathrm{BnTIQ}$ administration. Control rats were treated with the appropriate vehicle. Rats were sacrificed through decapitation at $2 \mathrm{~h}$ after the last drug injections, and different brain structures were dissected for further analysis. The experiments were performed between 9:00 and 16:00.
All experimental procedures were performed in accordance with the National Institutes of Health Guide for the Care and Use of Laboratory Animals and approved through the Bioethics Commission as compliant with Polish Law. All experimental procedures were approved through the Local Bioethics Commission of the Institute of Pharmacology, Polish Academy of Sciences in Kraków.

\section{Drugs}

1MeTIQ hydrochloride and 1BnTIQ hydrochloride were synthesized (according to Cannon and Webster 1958) at the Department of Drug Chemistry of the Institute of Pharmacology, the Polish Academy of Sciences in Krakow. The purity of the compound was verified by measuring the melting point, and homogeneity was assessed using a chromatographic column. The compounds were dissolved in a $0.9 \% \mathrm{NaCl}$ solution.

\section{Behavioral Study}

\section{Locomotor Activity}

Locomotor activity was assessed using actometers (OptoVarimex activity monitors; Columbus Inst., USA) linked on-line to a compatible IBM PC. Each cage $(43 \times 44 \times 25 \mathrm{~cm})$ perimeter was lined with an array of $15 \times 15$ photocell beams located $3 \mathrm{~cm}$ from the floor surface. Interruptions of the photocell beams were counted as a measure of horizontal locomotor activity and defined as the distance traveled (in $\mathrm{cm}$ ). The rats were administered an acute dose of 25 or $50 \mathrm{mg} / \mathrm{kg}$ (i.p.) 1MeTIQ (expression treatment group) or as the last dose (14-day chronic administration; development treatment group) at $20 \mathrm{~min}$ before the last dose of 1BnTIQ (25 mg/kg i.p.; 14-day chronic administration). Subsequently, the animals were transferred to experimental cages, and locomotor activity (horizontal activity, traveled distance in $\mathrm{cm}$ ) and rearing (vertical activity, time in sec) were recorded for $90 \mathrm{~min}$ and analyzed using the Auto-Track Software Program (Columbus Instruments, USA). Each group comprised seven animals.

\section{Biochemical Studies}

\section{Ex Vivo Experiments}

Dopamine Metabolism The rats were sacrificed through decapitation $2 \mathrm{~h}$ after the last 1BnTIQ injection, and the nucleus accumbens and striatum were immediately dissected. The tissue was frozen on dry ice $\left(\begin{array}{lll}-70 & \mathrm{C}\end{array}\right)$ until further use in a biochemical assay. The levels of dopamine (DA) and its metabolites, 3,4-dihydroxyphenylacetic acid 
(DOPAC), 3-methoxytyramine (3-MT), and homovanillic acid (HVA), were assayed through high-performance liquid chromatography (HPLC) with electrochemical detection (Hewlett Packard 1049A). The tissue samples were weighed and homogenized in ice-cold $0.1 \mathrm{M}$ perchloroacetic acid containing $0.05 \mathrm{mM}$ ascorbic acid. After centrifugation $(10,000 \mathrm{~g}$ for $5 \mathrm{~min})$, the supernatants were filtered through RC 58 0.2-im cellulose membranes (Bioanalytical Systems, West Lafayette, IN, USA). The HP 1050 chromatograph (Hewlett-Packard, Golden, CO, USA) was equipped with $\mathrm{C} 18$ columns. The electrochemical cell potential was $800 \mathrm{mV}$. The mobile phase comprised $0.05 \mathrm{M}$ citrate-phosphate buffer ( $\mathrm{pH} 3.5), 0.1 \mathrm{mM}$ EDTA, $1 \mathrm{mM}$ sodium octyl sulfonate, and $3.5 \%$ methanol. The flow rate was maintained at $1 \mathrm{ml} / \mathrm{min}$. Dopamine and its metabolites were quantified based on the chromatograph peak height compared with standards run on the day of analysis. Each group comprised seven animals.

Determination of Amino Acid Neurotransmitters The rats were sacrificed through decapitation $2 \mathrm{~h}$ after the last 1BnTIQ injection, and the frontal cortex was immediately dissected. The tissue was frozen on dry ice $\left(-70^{\circ} \mathrm{C}\right)$ until further use in a biochemical assay. The tissue was weighed, and subsequently homogenized in $4 \mathrm{ml}$ of chilled saline solution $(0.9 \%)$. The homogenate was centrifuged at $22.000 \mathrm{~g}$ for $10 \mathrm{~min}$ at $4{ }^{\circ} \mathrm{C}$. The supernatant was transferred and filtered through a $0.22-\mu \mathrm{m}$ pore size Millipore filter for derivatization. A $100-\mu$ l aliquot of mixed amino acid solution or sample supernatant, $175 \mu \mathrm{l}$ of borate buffer solution, $200 \mu \mathrm{l}$ of acetonitrile, and $25 \mu \mathrm{l}$ NBD-F working solution were mixed in a $1.5 \mathrm{ml}$ centrifuge tube. The well mixed solution was incubated in a $60^{\circ} \mathrm{C}$ water bath for 7 min in the dark. NBD-F reacts with amino group and enables temperature, $10 \mu \mathrm{l}$ of solution was injected into the equilibrated HPLC system. The analysis was performed on a Coulochem III HPLC system equipped with a UV DIONEX UltiMate 3000 detector $(472 \mathrm{~nm})$. The analyte was separated on an analytical Hypersil C18 column $(250 \mathrm{~mm}$ length $\times 4.6 \mathrm{~mm}, 5 \mu \mathrm{m}$ particle diameter) manufactured in Germany. The mobile phase comprised a mixture of acetonitrile-phosphate buffer $(0.02 \mathrm{~mol} / \mathrm{l}, \mathrm{pH} 6.0 ; 16: 84, \mathrm{v} / \mathrm{v})$ filtered through a $0.45-\mu \mathrm{m}$ nylon filter and degassed under ultrasound and vacuum for $30 \mathrm{~min}$. The mobile phase was delivered at a flow rate of $1.0 \mathrm{ml} / \mathrm{min}$, and the column temperature was set to $30{ }^{\circ} \mathrm{C}$.

Immunoblotting Protein was extracted through highspeed shaking in plastic tubes with stainless steel beads in a tissuelyser with $100 \mu \mathrm{l}$ of ice-cold RIPA lysis buffer (Sigma, USA), containing complete mini protease inhibitor (Roche Diagnostics, USA). After incubation for $30 \mathrm{~min}$, the homogenates were centrifuged at $10,000 \mathrm{~g}$ for $20 \mathrm{~min}$ at
$4{ }^{\circ} \mathrm{C}$.The resulting supernatants were collected and subjected to protein analysis using the Bicinchoninic Acid Assay Kit (Sigma, USA). Equal amounts of protein extracts $(12 \mu \mathrm{g})$ were boiled in Laemmli buffer containing $5 \% \beta$ mercaptoethanol for $5 \mathrm{~min}$, separated through SDS-PAGE (4-15\%), and transferred to nitrocellulose membranes. The membranes were blocked with $5 \%$ non-fat dry milk in Tris-buffered saline containing Tween-20 (TBST; $\mathrm{pH}=7.6$ ) for $1 \mathrm{~h}$ at room temperature and incubated with a primary antibody against tyrosine hydroxylase $(1: 2,000$; Cell Signaling, USA) or $\alpha$-synuclein $(1: 2,000$; Cell Signaling, USA) overnight at $4{ }^{\circ} \mathrm{C}$. After three washes with blocking solution, the membranes were incubated with the appropriate secondary antibodies for $1 \mathrm{~h}$ at room temperature, followed by three washes with TBST. Antibody binding was detected using an enhanced chemiluminescence kit (ECL Plus, Pierce, USA). Equal loading proteins were further confirmed after probing with anti-calnexin antiserum (CNX; 1:5,000; Enzo Life Sciences, USA) or anti- $\beta$-actin antiserum (1:5,000; Sigma, USA). All Western blot analyses were performed at least twice to confirm the results. The chemiluminescence signal was visualized using a Luminescent Image Analyzer Fuji-Las 4000 (Fuji, Japan). Immunoreactive bands were quantified using an image analyzer (ScienceLab, MultiGauge V3.0).

\section{Calculations and Statistics}

Two-way analysis of variance (ANOVA) for repeated measures was used to analyze the results of the behavioral test (locomotor activity). Differences between the control and experimental groups were assessed using Duncan's post hoc test.

The comparison of the effects of acute and multiple administration of 1BnTIQ and the effect of chronic 1MeTIQ administration on 1BnTIQ-produced changes in the concentration of EAA were analyzed by one-way ANOVA, followed by Duncan's post hoc test. The results of the biochemical experiments were analyzed using twoway ANOVA, followed by Duncan's post hoc test, when appropriate. These indices were calculated using the concentrations of individual tissue samples $(n=6-7)$.

\section{Results}

\section{Behavioral Study}

The Effect of Acute 1MeTIQ Administration on 1BnTIQInduced Hyperactivity in Rats

The acute administration of $1 \mathrm{MeTIQ}$ (50 mg/kg i.p.) induced a significant reduction in the horizontal 
exploratory activity of rats $(P<0.01)$. In addition, the chronic administration of 1BnTIQ $(25 \mathrm{mg} / \mathrm{kg}$ i.p.) produced a significant $(P<0.05)$ elevation of exploratory activity (Fig. 1a). In the mixed group, an acute dose of 1MeTIQ completely antagonized 1BnTIQ-induced hyperactivity.

The Effect of Chronic 1MeTIQ Administration on IBnTIQInduced Hyperactivity in Rats

As shown in Fig. 1b, the chronic administration of 1MeTIQ (50 mg/kg i.p.) over 14 days induced a significant $(P<0.01)$ decrease in exploratory activity. The multiple (14-day) administration of 1BnTIQ at a low dose of $25 \mathrm{mg} /$ $\mathrm{kg}$ (i.p.) significantly increased $(P<0.05)$ the exploratory activity during the first $30 \mathrm{~min}$ of measurement. In the mixed group, 1MeTIQ completely antagonized 1BnTIQinduced hyperactivity (Fig. 1b).
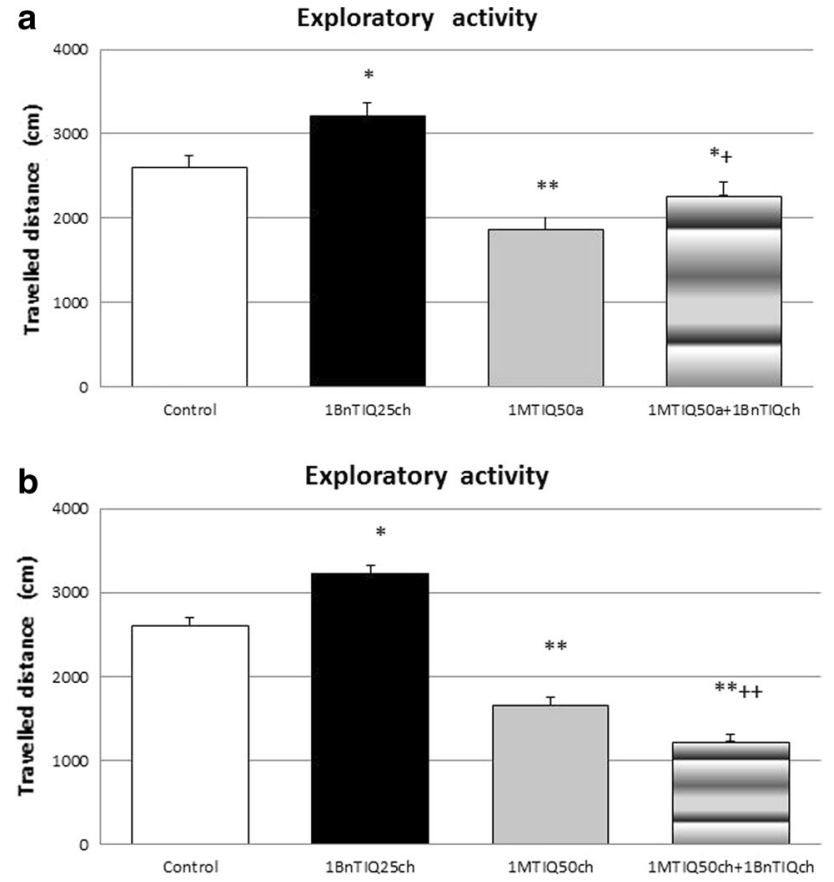

Fig. 1 The influence of acute (a) and chronic (b) treatment with $1 \mathrm{MeTIQ}$ on changes in exploratory activity induced by chronic administration of 1BnTIQ. 1BnTIQ (25 mg/kg i.p.) was administered chronic during 14 consecutive days. 1MeTIQ (50 mg/kg i.p) was given acute (a) or chronic during 14 consecutive days (b). In the mixed group, 1MeTIQ was injected 20 min before 1BnTIQ administration. A control group was treated by saline. Rats were placed into actometers immediately after last drugs administration. Movements were recorded for $30 \mathrm{~min}$. The data are expressed as the mean$\mathrm{s} \pm$ SEM ( $n=7$ animals). Data were analyzed with a two-way ANOVA for repeated measures, followed by Duncan's post hoc test. Statistical significance: $* P<0.05$; ** $P<0.01$ versus saline-treated group; ${ }^{+} P<0.05$ versus 1 BnTIQ-treated group

\section{Biochemical Studies}

\section{Ex Vivo Experiments}

The Comparison of the Effects of the Acute and Multiple Administration of 1 BnTIQ on Dopamine Metabolism in Rat Brain Structures One-way ANOVA demonstrated a significant effect of 1BnTIQ treatment $(F[2,18]=18.61$, $P<0.01$ ) on the level of DA in the striatum (Table 1). Duncan's post hoc analysis demonstrated that both the acute and multiple administration of 1BnTIQ significantly decreased the level of DA (respectively, 40 and $25 \%$; $P<0.01)$. The statistical analysis indicated no effect of 1BnTIQ treatment $(F[2,18]=1.31$, N.S. $)$ on DOPAC concentration (Table 1). One-way ANOVA demonstrated a significant effect of 1BnTIQ treatment $(F[2,18]=5.81$, $P<0.05$ ) on the level of 3-MT in the striatum (Table 1). The post hoc analysis showed that the acute injection of 1BnTIQ reduced the concentration of 3-MT in the rat striatum (approximately $25 \%, P<0.05$ ). The statistical analysis indicated a significant effect of 1BnTIQ treatment $(F[2,18]=4.81, P<0.05)$ on the HVA concentration (Table 1). Duncan's post hoc analysis demonstrated that the acute administration of 1BnTIQ significantly increased the level of HVA (approximately $60 \% ; P<0.05$ ).

One-way ANOVA indicated a significant effect of 1BnTIQ treatment $(F[2,18]=18.73, P<0.01)$ on the level of DA in the nucleus accumbens (Table 1). The post hoc test showed that both the acute and multiple administration of 1BnTIQ significantly decreased the level of DA (respectively, 40 and $30 \% ; P<0.01$ ). Treatment with 1BnTIQ $(F[2,18]=7.64, P<0.01)$ significantly affected the DOPAC concentration in the nucleus accumbens (Table 1). Duncan's post hoc analysis demonstrated that the multiple administration of 1BnTIQ significantly decreased the level of DOPAC (approximately $25 \%$; $P<0.01)$. One-way ANOVA indicated a significant effect of 1BnTIQ treatment $(F[2,18]=10.26, P<0.01)$ on the 3 -MT concentration in the nucleus accumbens (Table 1). The post hoc test showed that both acute and multiple administration of 1BnTIQ significantly decreased the level of 3-MT (respectively 40 and $30 \% ; P<0.01$ ). The statistical analysis indicated a significant effect of $1 \mathrm{BnTIQ}$ treatment $(F[2,18]=4.81, P<0.05)$ on the HVA concentration (Table 1). Duncan's post hoc analysis demonstrated that the acute administration of 1BnTIQ significantly increased the level of HVA (approximately $20 \% ; P<0.05)$.

The Effect of Acute 1MeTIQ Administration on Changes in Dopamine Metabolism Induced After the Chronic Administration of IBnTIQ in Rat Brain Structures Table 2 Striatum 
Table 1 The comparison of the influence of acute and chronic administration of 1BnTIQ on dopamine (DA) metabolism in rat brain structures

\begin{tabular}{|c|c|c|c|c|c|}
\hline Treatment & $N$ & $\begin{array}{l}\text { DA } \\
\mathrm{ng} / \mathrm{g}\end{array}$ & $\begin{array}{l}\text { DOPAC } \\
\mathrm{ng} / \mathrm{g}\end{array}$ & $\begin{array}{l}\text { 3-MT } \\
\mathrm{ng} / \mathrm{g}\end{array}$ & $\begin{array}{l}\text { HVA } \\
\text { ng/g }\end{array}$ \\
\hline \multicolumn{6}{|l|}{ Striatum } \\
\hline Saline & 7 & $9894 \pm 618$ & $2158 \pm 113$ & $416 \pm 30$ & $1027 \pm 90$ \\
\hline 1BnTIQ/25-1× & 7 & $5817 \pm 415^{* *}$ & $1897 \pm 83$ & $316 \pm 14^{* *}$ & $1638 \pm 171^{* *}$ \\
\hline 1BnTIQ/25-14x & 7 & $7416 \pm 356^{* *+}$ & $1999 \pm 142$ & $388 \pm 16^{+}$ & $1383 \pm 146$ \\
\hline$F$ & & $\begin{array}{l}F_{(2 / 18)}=18.61 \\
P<0.00004\end{array}$ & $\begin{array}{l}F_{(2 / 18)}=1.31 \\
\mathrm{NS}\end{array}$ & $\begin{array}{l}F_{(2 / 18)}=5.81 \\
P<0.01\end{array}$ & $\begin{array}{l}F_{(2 / 18)}=4.81 \\
P<0.02\end{array}$ \\
\hline \multicolumn{6}{|l|}{ Nucleus accumbens } \\
\hline Saline & 7 & $9.089 \pm 406$ & $2.742 \pm 98$ & $320 \pm 19$ & $1.049 \pm 69$ \\
\hline 1BnTIQ/25-1× & 7 & $5.656 \pm 505^{* *}$ & $2.408 \pm 180$ & $195 \pm 24 * *$ & $1.245 \pm 141 *$ \\
\hline 1BnTIQ/25-14× & 7 & $6.172 \pm 358^{* *}$ & $2.024 \pm 94 * *+$ & $233 \pm 16^{* *}$ & $926 \pm 53^{+}$ \\
\hline$F$ & & $\begin{array}{l}F_{(2 / 18)}=18.73 \\
P<0.00004\end{array}$ & $\begin{array}{l}F_{(2 / 18)}=7.64 \\
P<0.003\end{array}$ & $\begin{array}{l}F_{(2 / 18)}=10.26 \\
P<0.001\end{array}$ & $\begin{array}{l}F_{(2 / 18)}=4.81 \\
P<0.02\end{array}$ \\
\hline
\end{tabular}

1BnTIQ (25 mg/kg i.p.) was administered acute or chronically during 14 consecutive days. Rats were decapitated $2 \mathrm{~h}$ after the injections. The results are expressed as the means \pm SEM of seven samples ( $n=7$ animals per group). Data were analyzed with a one-way ANOVA followed by Duncan's post hoc test. Statistical significance: $* P<0.05$; $* * P<0.01$ versus Saline group; ${ }^{+} P<0.05$ versus acute 1BnTIQ group
Two-way ANOVA indicated a significant effect of chronic treatment with 1BnTIQ $(F[2,35]=29.41, P<0.01)$ and an acute dose of 1MeTIQ $(F[2,35]=8.88, P<0.01)$ on the DA concentration in the striatum (Table 2). The interaction between 1BnTIQ and 1MeTIQ treatment was not significant $(F[2,35]=0.79$, N.S. $)$. Duncan's post hoc analysis demonstrated that the multiple administration of 1BnTIQ significantly decreased the level of DA (approximately $25 \% ; P<0.01)$. In contrast, 1MeTIQ co-administered with the last dose of 1BnTIQ completely antagonized the 1BnTIQ-induced reduction in DA (the DA level remained similar to the control level) (Table 2).

Two-way ANOVA demonstrated a significant effect of 1BnTIQ $\quad(F[2,35]=5.11, \quad P<0.05)$ and 1 MeTIQ $(F[2,35]=64.73, P<0.01)$ on the DOPAC concentration in the striatum (Table 2). The interaction between 1BnTIQ and 1MeTIQ was not significant $(F[2,35]=0.01$, N.S. $)$. The post hoc analysis indicated that the acute administration of 1MeTIQ (at both doses examined) decreased the DOPAC concentration (approximately 30 and $45 \%$; $P<0.01)$ and that this effect was enhanced in the mixed groups (approximately 40 and $55 \%$ ) (Table 2).

The statistical analysis showed no effect of 1 BnTIQ $(F[2,35]=0.5$, N.S. $)$ or the interaction between 1BnTIQ and 1MeTIQ $(F[2,35]=0.09 ;$ N.S. $)$ on the levels of 3-MT in the rat striatum. In contrast, the effect of $1 \mathrm{MeTIQ}$ was significant $(F[2,35]=36.49, P<0.01)$ (Table 2). Duncan's post hoc test showed that both doses of 1MeTIQ administered alone increased the 3-MT concentration (respectively, 50 and $70 \%, P<0.01$ ), and similar effects were observed in the mixed groups (Table 2).
Treatment with 1BnTIQ $(F[2,35]=20.97, P<0.01)$ and 1MeTIQ $(F[2,35]=18.87, P<0.01)$ significantly affected the HVA concentration in the striatum (Table 2). This analysis also revealed a significant interaction between 1BnTIQ and 1MeTIQ $(F[2,35]=14.88$, $P<0.01)$. The post hoc analysis showed that the chronic administration of 1BnTIQ significantly increased the level of HVA (approx. $35 \%, P<0.05$ ), and this effect was completely blocked through the administration of both concentrations of 1MeTIQ (Table 2).

Two-way ANOVA demonstrated that chronic treatment with 1BnTIQ significantly increased the rate of DA oxidation, measured as index [DOPAC]/[DA] $(P<0.01)$. This effect was completely inhibited through the acute administration of 1MeTIQ at both doses examined (Fig. 2a). Similarly, the rate of total DA catabolism (measured as index [HVA]/[DA]) was significantly $(P<0.01)$ increased after the multiple administration of $1 \mathrm{BnTIQ}$, and this effect was antagonized with a single dose of 1MeTIQ (Fig. 2b).

\section{Nucleus Accumbens}

Two-way ANOVA indicated a significant effect of both 1BnTIQ $\quad(F[2,35]=17.97, \quad P<0.01) \quad$ and $1 \mathrm{MeTIQ}$ $(F[2,35]=4.4, P<0.01)$ treatments on the DA concentration in the nucleus accumbens (Table 2). In addition, the interaction between 1BnTIQ and 1MeTIQ was not significant $(F[2,35]=1.17$, N.S. $)$. Duncan's post hoc test showed that the chronic administration of 1BnTIQ 
Table 2 The influence of acute administration of 1MeTIQ on changes induced by chronic treatment with 1BnTIQ in the concentration of dopamine (DA) and its metabolites

\begin{tabular}{|c|c|c|c|c|c|c|}
\hline \multicolumn{2}{|l|}{ Treatment } & \multirow[t]{2}{*}{$N$} & \multirow[t]{2}{*}{ DA (ng/g) } & \multirow[t]{2}{*}{ DOPAC (ng/g) } & \multirow[t]{2}{*}{ 3-MT (ng/g) } & \multirow[t]{2}{*}{ HVA (ng/g) } \\
\hline Acute & Chronic & & & & & \\
\hline \multicolumn{7}{|l|}{ Striatum } \\
\hline Saline & Saline & 7 & $9894 \pm 618$ & $2158 \pm 113$ & $416 \pm 30$ & $1027 \pm 90$ \\
\hline 1MeTIQ/25 & Saline & 7 & $11055 \pm 264$ & $1432 \pm 61^{* *}$ & $620 \pm 34 * *$ & $288 \pm 27 * *$ \\
\hline 1MeTIQ/50 & Saline & 7 & $10979 \pm 502$ & $1144 \pm 47^{* *}$ & $706 \pm 38 * *$ & $1049 \pm 87$ \\
\hline Saline & MeTIQ/25 & 6 & $7416 \pm 356^{* *}$ & $1999 \pm 142$ & $388 \pm 16$ & $1383 \pm 146^{*}$ \\
\hline 1MeTIQ/25 & 1BnTIQ/25 & 7 & $9649 \pm 542^{++}$ & $1254 \pm 64 * *++$ & $587 \pm 45^{* *++}$ & $1072 \pm 58^{+}$ \\
\hline 1MeTIQ/25 & 1BnTIQ/25 & 7 & $9105 \pm 118^{++}$ & $968 \pm 79 * *++$ & $703 \pm 44 * *++$ & $881 \pm 60^{++}$ \\
\hline Effect of 1MeTIQ & & & $\begin{array}{l}F_{(2 / 35)}=8.88 \\
P<0.0007\end{array}$ & $\begin{array}{l}F_{(2 / 35)}=64.73 \\
P<0.0000001\end{array}$ & $\begin{array}{l}F_{(2 / 35)}=36.49 \\
P<0.0000001\end{array}$ & $\begin{array}{l}F_{(2 / 35)}=18.87 \\
P<0.000003\end{array}$ \\
\hline Effect of 1BnTIQ & & & $\begin{array}{l}F_{(2 / 35)}=29.41 \\
P<0.00004\end{array}$ & $\begin{array}{l}F_{(2 / 35)}=5.11 \\
P<0.03\end{array}$ & $\begin{array}{l}F_{(2 / 35)}=0.50 \\
\mathrm{NS}\end{array}$ & $\begin{array}{l}F_{(2 / 35)}=20.97 \\
P<0.00005\end{array}$ \\
\hline Interaction of $1 \mathrm{MeTIQ}+1 \mathrm{BnTIQ}$ & & & $\begin{array}{l}F_{(2 / 35)}=0.79 \\
\mathrm{NS}\end{array}$ & $\begin{array}{l}F_{(2 / 35)}=0.01 \\
\mathrm{NS}\end{array}$ & $\begin{array}{l}F_{(2 / 35)}=0.09 \\
\mathrm{NS}\end{array}$ & $\begin{array}{l}F_{(2 / 35)}=14.88 \\
P<0.00002\end{array}$ \\
\hline \multicolumn{7}{|l|}{ Nucleus accumbens } \\
\hline Saline & Saline & 7 & $9089 \pm 406$ & $2742 \pm 98$ & $320 \pm 19$ & $1049 \pm 69$ \\
\hline 1MeTIQ/25 & Saline & 7 & $9796 \pm 388$ & $1742 \pm 89 * *$ & $386 \pm 18$ & $901 \pm 71$ \\
\hline 1MeTIQ/50 & Saline & 7 & $9765 \pm 1145$ & $1406 \pm 204 * *$ & $402 \pm 47$ & $854 \pm 129$ \\
\hline Saline & 1BnTIQ/25 & 6 & $6172 \pm 358 * *$ & $2024 \pm 94 * *$ & $233 \pm 16^{*}$ & $926 \pm 53$ \\
\hline 1MeTIQ/25 & 1BnTIQ/25 & 7 & $8316 \pm 413^{+}$ & $1378 \pm 85^{* *++}$ & $373 \pm 24^{++}$ & $693 \pm 44^{* *+}$ \\
\hline 1MeTIQ/50 & 1BnTIQ/25 & 7 & $8351 \pm 476^{+}$ & $1080 \pm 95^{* *++}$ & $467 \pm 34^{* *++}$ & $664 \pm 56^{* *+}$ \\
\hline Effect of 1MeTIQ & & & $\begin{array}{l}F_{(2 / 35)}=4.40 \\
P<0.01\end{array}$ & $\begin{array}{l}F_{(2 / 35)}=54.39 \\
P<0.0000001\end{array}$ & $\begin{array}{l}F_{(2 / 35)}=17.01 \\
P<0.000007\end{array}$ & $\begin{array}{l}F_{(2 / 35)}=5.80 \\
P<0.006\end{array}$ \\
\hline Effect of 1BnTIQ & & & $\begin{array}{l}F_{(2 / 35)}=17.97 \\
P<0.0001\end{array}$ & $\begin{array}{l}F_{(2 / 35)}=25.95 \\
P<0.00001\end{array}$ & $\begin{array}{l}F_{(2 / 35)}=0.27 \\
\mathrm{NS}\end{array}$ & $\begin{array}{l}F_{(2 / 35)}=8.75 \\
P<0.005\end{array}$ \\
\hline Interaction of $1 \mathrm{MeTIQ}+1 \mathrm{BnTIQ}$ & & & $\begin{array}{l}F_{(2 / 35)}=1.17 \\
\mathrm{NS}\end{array}$ & $\begin{array}{l}F_{(2 / 35)}=1.86 \\
\mathrm{NS}\end{array}$ & $\begin{array}{l}F_{(2 / 35)}=3.74 \\
P<0.03\end{array}$ & $\begin{array}{l}F_{(2 / 35)}=0.19 \\
\mathrm{NS}\end{array}$ \\
\hline
\end{tabular}

1BnTIQ was chronically administered (25 mg/kg i.p.) during 14 consecutive days. In the mixed group, $1 \mathrm{MeTIQ}$ (50 mg/kg i.p.) was given once, $20 \mathrm{~min}$ before last $1 \mathrm{BnTIQ}$ administration. Rats were decapitated $2 \mathrm{~h}$ after the injections. The results are expressed as the means \pm SEM of six to seven samples ( $n=6-7$ animals per group). Data were analyzed with a two-way ANOVA followed by Duncan's post hoc test. Statistical significance: $* P<0.05 ; * * P<0.01$ versus Saline/Saline group; ${ }^{+} P<0.05,{ }^{++} P<0.01$ versus 1 BnTIQ group

decreased the DA concentration (approx. $30 \%, P<0.01$ ). Both concentrations of 1 MeTIQ partially antagonized this effect (Table 2).

The statistical analysis showed a significant effect of 1BnTIQ $\quad(F[2,35]=25.95, \quad P<0.01) \quad$ and 1 MeTIQ $(F[2,35]=54.39, P<0.01)$ treatments on the DOPAC levels. In contrast, the interaction between 1BnTIQ and 1MeTIQ $(F[2,35]=1.86$, N.S. $)$ was not significant (Table 2). The post hoc analysis demonstrated a reduction in the level of DOPAC through chronic treatment with 1BnTIQ (approximately $25 \%$ ), and both doses of 1MeTIQ (respectively, 40 and $50 \%$ ). In the mixed groups, this reduction was markedly enhanced (respectively, 50 and $60 \%$ ). (Table 2).

Two-way ANOVA further revealed no effect of the chronic administration of 1BnTIQ $(F[2,35]=0.27$, N.S. $)$ on the 3-MT concentration in the nucleus accumbens (Table 2). However, the effects of 1MeTIQ $(F[2,35]=$ 17.01, $P<0.01)$ and the interaction between 1BnTIQ and 1MeTIQ were significant $(F[2,35]=3.74, \quad P<0.05)$. Duncan's post hoc test showed that chronic treatment with 1BnTIQ ( $25 \mathrm{mg} / \mathrm{kg}$ ) reduced the levels of 3-MT (approximately $30 \%$ ), and this effect was completely blocked using both doses of 1MeTIQ (Table 2).The statistical analysis indicated a significant effect of 1BnTIQ $(F[2,35]=8.75, P<0.01)$ and 1MeTIQ $(F[2,35]=5.80$, $P<0.01)$ treatments on the HVA levels, but the interaction between 1BnTIQ and 1MeTIQ $(F[2,35]=0.19$, N.S. $)$ was not significant. The post hoc analysis showed that treatment with 1BnTIQ concomitant with 1MeTIQ significantly reduced the HVA levels (approximately 35\%) (Table 2). 
Fig. 2 The influence of acute (a) and chronic

(b) administration of 1MeTIQ on the elevation rate of dopamine oxidation induced by chronic treatment with 1BnTIQ. 1BnTIQ (25 mg/kg i.p.) was administered chronic during 14 consecutive days. 1MeTIQ (25 or $50 \mathrm{mg} / \mathrm{kg}$ i.p) was given acute (a) or chronic during 14 consecutive days (b). In the mixed group, 1MeTIQ was injected 20 min before $1 \mathrm{BnTIQ}$ administration. A control group was treated by saline. Rats were decapitated $2 \mathrm{~h}$ after the injections. The data are expressed as the means \pm SEM ( $n=6-7$ animals). Data were analyzed with a two-way ANOVA, followed by Duncan's post hoc test. Statistical significance: $* P<0.05$; ** $P<0.01$ versus salinetreated group; ${ }^{++} P<0.01$ versus 1BnTIQ-treated group a [DOPAC]/[DA] STRIATUM

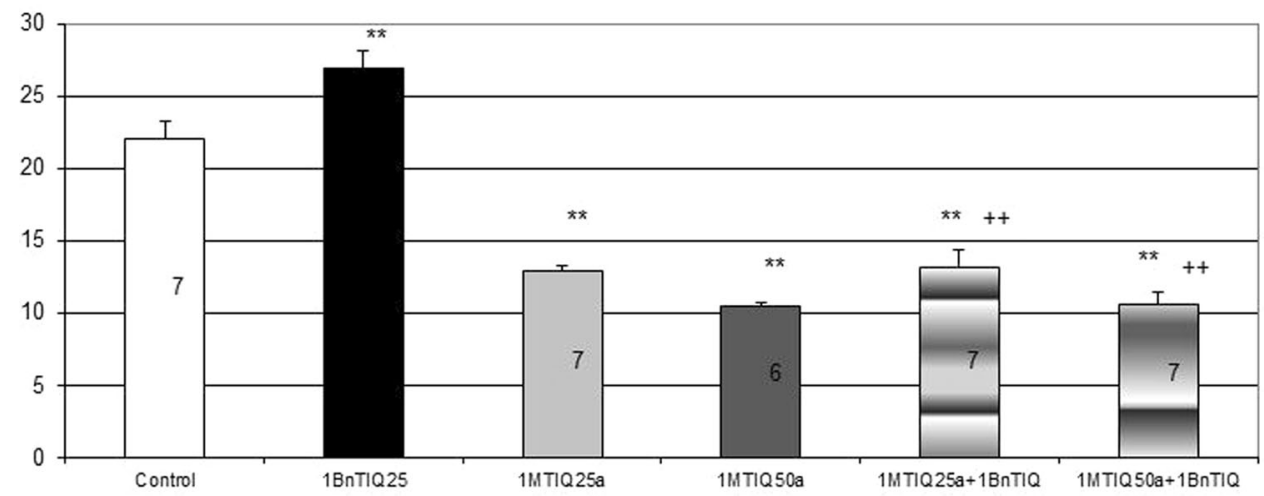

b $[\mathrm{DOPAC}] /[\mathrm{DA}]$

STRIATUM

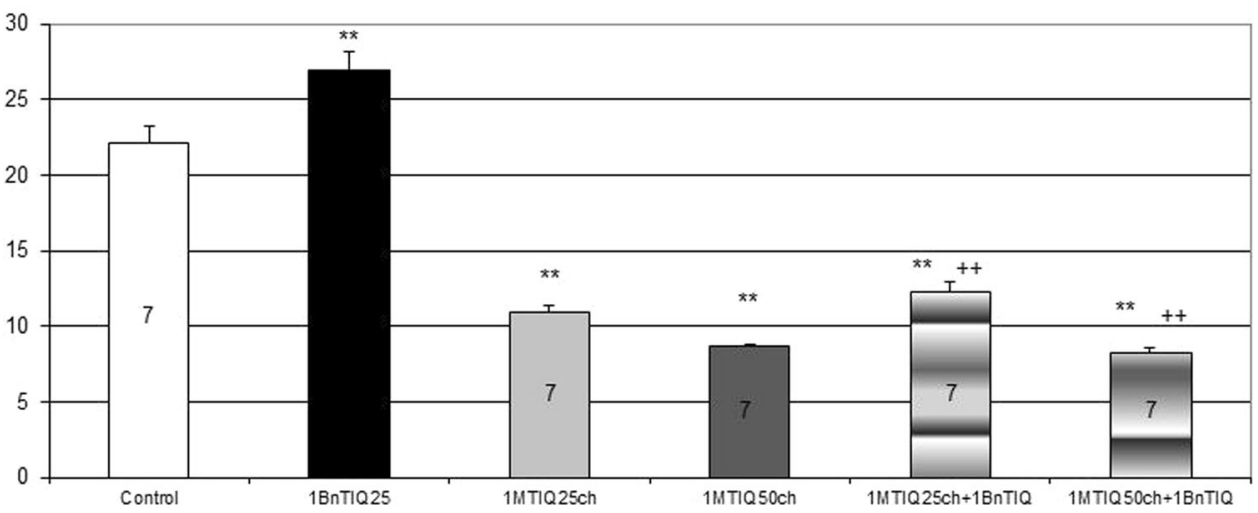

The Effect of Chronic 1MeTIQ Administration on Changes in Dopamine Metabolism Induced Through the Chronic Administration of $1 B n T I Q$ in Rat Brain Structures Table 3 Striatum

Two-way ANOVA revealed a significant effect of chronic treatment with 1BnTIQ $(F[2,36]=41.37, P<0.01)$ and the chronic administration of 1 MeTIQ $(F[2,36]=10.18$, $P<0.01)$ on the DA concentration in the striatum (Table 3). There was no significant interaction between the chronic administration of 1BnTIQ and multiple injections with 1 MeTIQ $(F[2,36]=2.01$, N.S. $)$. Duncan's post hoc test showed that the chronic administration of $1 \mathrm{BnTIQ}$ reduced the DA concentration (approximately $25 \%$; $P<0.01$ ), while the multiple administration of $1 \mathrm{MeTIQ}$ (in both doses) increased neurotransmitter (by $20 \%$; $P<0.05)$. Additionally, when the chronic administration of 1 BnTIQ (25 mg/kg) was coupled with the chronic administration of $1 \mathrm{MeTIQ}$, the concentration of dopamine returned to the control level (Table 3).

Two-way ANOVA demonstrated a significant effect of chronic treatment with 1BnTIQ $(F[2,36]=6.49$, $P<0.01)$ and the multiple administration of $1 \mathrm{MeTIQ}$
$(F[2,36]=104.27, P<0.01)$ on the DOPAC concentration in the striatum (Table 3). Notably, the interaction between 1BnTIQ and 1MeTIQ was not significant $(F[2,36]=0.11$, N.S. $)$. The post hoc analysis showed that chronic 1MeTIQ administration strongly decreased the DOPAC concentration (respectively, 40 and $55 \%$; $P<0.01)$; this effect was enhanced in the mixed group (respectively, 55 and $65 \% ; P<0.01$ ) (Table 3).

The statistical analysis revealed no effect of chronic treatment with 1BnTIQ $(F[2,36]=0.48$, N.S. $)$ on the level of 3-MT (Table 3). Chronic treatment with 1MeTIQ $(F[2,36]=70.6, P<0.01)$ significantly affected the $3-\mathrm{MT}$ concentration. There was no interaction between the chronic administration of 1BnTIQ and the multiple administration of 1 MeTIQ $(F[2,36]=0.41$, N.S. $)$. Duncan's post hoc test showed that chronic treatment with 1 MeTIQ (in both doses) increased the 3-MT concentration (approximately 145 and $180 \% ; P<0.01$ ). A similar effect was observed with the chronic administration of 1BnTIQ $(25 \mathrm{mg} / \mathrm{kg})$ coupled with the chronic administration of 1MeTIQ, (Table 3).

Two-way ANOVA demonstrated no effect of chronic 1BnTIQ treatment $(F[2,36]=1.18$, N.S. $)$ on the HVA 
Table 3 The influence of chronic administration of 1MeTIQ on changes induced by chronic treatment with 1BnTIQ in the concentration of dopamine (DA) and its metabolites

\begin{tabular}{|c|c|c|c|c|c|c|}
\hline \multicolumn{2}{|l|}{ Treatment } & \multirow[t]{2}{*}{$N$} & \multirow[t]{2}{*}{ DA (ng/g) } & \multirow[t]{2}{*}{ DOPAC (ng/g) } & \multirow[t]{2}{*}{ 3-MT (ng/g) } & \multirow[t]{2}{*}{ HVA (ng/g) } \\
\hline Chronic & Chronic & & & & & \\
\hline \multicolumn{7}{|l|}{ Striatum } \\
\hline Saline & Saline & 7 & $9894 \pm 618$ & $2158 \pm 113$ & $416 \pm 30$ & $1027 \pm 90$ \\
\hline 1MeTIQ/25 & Saline & 7 & $11441 \pm 254^{*}$ & $1255 \pm 59^{* *}$ & $603 \pm 17 * *$ & $930 \pm 47$ \\
\hline 1MeTIQ/50 & Saline & 7 & $11259 \pm 579^{*}$ & $976 \pm 55^{* *}$ & $737 \pm 35^{* *}$ & $868 \pm 48$ \\
\hline Saline & 1BnTIQ/25 & 7 & $7416 \pm 356^{* *}$ & $1999 \pm 142$ & $388 \pm 16$ & $1383 \pm 146^{* *}$ \\
\hline 1MeTIQ/25 & 1BnTIQ/25 & 7 & $8390 \pm 239^{*}$ & $1029 \pm 49^{* *++}$ & $616 \pm 19^{* *++}$ & $853 \pm 59^{++}$ \\
\hline 1MeTIQ/50 & 1BnTIQ/25 & 7 & $9924 \pm 416^{++}$ & $823 \pm 51^{* *++}$ & $705 \pm 37^{* *++}$ & $807 \pm 53^{++}$ \\
\hline Effect of $1 \mathrm{MeTIQ}$ & & & $\begin{array}{l}F_{(2 / 36)}=10.18 \\
P<0.0003\end{array}$ & $\begin{array}{l}F_{(2 / 36)}=104.27 \\
P<0.0000001\end{array}$ & $\begin{array}{l}F_{(2 / 36)}=70.60 \\
P<0.0000001\end{array}$ & $\begin{array}{l}F_{(2 / 36)}=11.65 \\
P<0.0001\end{array}$ \\
\hline Effect of 1BnTIQ & & & $\begin{array}{l}F_{(2 / 36)}=41.37 \\
P<0.0000001\end{array}$ & $\begin{array}{l}F_{(2 / 36)}=6.49 \\
P<0.01\end{array}$ & $\begin{array}{l}F_{(2 / 36)}=0.48 \\
\text { NS }\end{array}$ & $\begin{array}{l}F_{(2 / 36)}=1.18 \\
\mathrm{NS}\end{array}$ \\
\hline Interaction of $1 \mathrm{MeTIQ}+1 \mathrm{BnTIQ}$ & & & $\begin{array}{l}F_{(2 / 36)}=2.01 \\
\text { NS }\end{array}$ & $\begin{array}{l}F_{(2 / 36)}=0.108 \\
\text { NS }\end{array}$ & $\begin{array}{l}F_{(2 / 36)}=0.41 \\
\text { NS }\end{array}$ & $\begin{array}{l}F_{(2 / 36)}=4.47 \\
P<0.01\end{array}$ \\
\hline \multicolumn{7}{|l|}{ Nucleus accumbens } \\
\hline Saline & Saline & 7 & $9089 \pm 406$ & $2742 \pm 98$ & $320 \pm 19$ & $1049 \pm 69$ \\
\hline 1MeTIQ/25 & Saline & 7 & $9552 \pm 669$ & $1295 \pm 105^{* *}$ & $354 \pm 25$ & $689 \pm 79 * *$ \\
\hline 1MeTIQ/50 & Saline & 7 & $8892 \pm 363$ & $1050 \pm 60 * *$ & $405 \pm 29^{*}$ & $593 \pm 33 * *$ \\
\hline Saline & 1BnTIQ/25 & 7 & $6172 \pm 358 * *$ & $2024 \pm 94 * *$ & $233 \pm 16^{*}$ & $926 \pm 53$ \\
\hline 1MeTIQ/25 & 1BnTIQ/25 & 7 & $6971 \pm 373 * *++$ & $1038 \pm 74^{* *++}$ & $369 \pm 22^{++}$ & $518 \pm 43^{* *++}$ \\
\hline 1MeTIQ/50 & 1BnTIQ/25 & 7 & $8442 \pm 581^{++}$ & $871 \pm 77^{* *++}$ & $393 \pm 28^{*++}$ & $509 \pm 24^{* *++}$ \\
\hline Effect of 1MeTIQ & & & $\begin{array}{l}F_{(2 / 36)}=2.43 \\
\mathrm{NS}\end{array}$ & $\begin{array}{l}F_{(2 / 36)}=159.28 \\
P<0.0000001\end{array}$ & $\begin{array}{l}F_{(2 / 36)}=14.17 \\
P<0.00002\end{array}$ & $\begin{array}{l}F_{(2 / 36)}=39.26 \\
P<0.0000001\end{array}$ \\
\hline Effect of 1BnTIQ & & & $\begin{array}{l}F_{(2 / 36)}=26.21 \\
P<0.00001\end{array}$ & $\begin{array}{l}F_{(2 / 36)}=29.92 \\
P<0.000004\end{array}$ & $\begin{array}{l}F_{(2 / 36)}=2.05 \\
\text { NS }\end{array}$ & $\begin{array}{l}F_{(2 / 36)}=8.25 \\
P<0.006\end{array}$ \\
\hline Interaction of $1 \mathrm{MeTIQ}+1 \mathrm{BnTIQ}$ & & & $\begin{array}{l}F_{(2 / 36)}=3.98 \\
P<0.02\end{array}$ & $\begin{array}{l}F_{(2 / 36)}=5.74 \\
P<0.006\end{array}$ & $\begin{array}{l}F_{(2 / 36)}=2.48 \\
\text { NS }\end{array}$ & $\begin{array}{l}F_{(2 / 36)}=0.33 \\
\mathrm{NS}\end{array}$ \\
\hline
\end{tabular}

1BnTIQ was chronically administered ( $25 \mathrm{mg} / \mathrm{kg}$ i.p.) during 14 consecutive days. In the mixed group, $1 \mathrm{MeTIQ}$ (50 mg/kg i.p.) was given also chronically, $20 \mathrm{~min}$ before each 1 BnTIQ administration. Rats were decapitated $2 \mathrm{~h}$ after the injections. The results are expressed as the means \pm SEM of seven samples ( $n=7$ animals per group). Data were analyzed with a two-way ANOVA followed by Duncan's post hoc test. Statistical significance: $* P<0.05$; ** $P<0.01$ versus Saline/Saline group; ${ }^{+} P<0.05$; ${ }^{++} P<0.01$ versus 1 BnTIQ group

concentration in the striatum (Table 3). In contrast, the chronic administration of $1 \operatorname{MeTIQ}(F[2,36]=11.65$, $P<0.01)$ significantly affected the level of HVA. An interaction between 1BnTIQ and 1MeTIQ was also detected $(F[2,36]=4.47, P<0.01)$. The post hoc analysis showed that 1BnTIQ increased the HVA concentration (approximately $135 \%$; $P<0.01$ ); this effect was completely blocked through chronic treatment with both concentrations of 1MeTIQ (Table 3).

Two-way ANOVA indicated that repeated treatment with 1BnTIQ significantly increased the rate of DA oxidation measured as index [DOPAC]/[DA] $(P<0.01)$. This effect was completely inhibited through the chronic administration of 1MeTIQ at both doses examined (Fig. 3a). Similarly, the rate of total DA catabolism (measured as index $[\mathrm{HVA}] /[\mathrm{DA}]$ ) was significantly
$(P<0.01)$ increased through the multiple administration of $1 \mathrm{BnTIQ}$, and this effect was antagonized through the chronic administration of 1MeTIQ (Fig. 3b).

\section{Nucleus Accumbens}

Two-way ANOVA revealed a significant effect of chronic 1BnTIQ treatment $(F[2,36]=26.21, P<0.01)$ on the DA concentration in the nucleus accumbens (Table 3 ). In contrast, multiple 1MeTIQ administration produced no effect on the level of DA $(F[2,36]=2.43$, N.S.). The statistical analysis revealed a significant interaction between the chronic administration of 1BnTIQ and 1MeTIQ $(F[2,36]=3.98, P<0.05)$. The post hoc test indicated that the multiple injection of 1BnTIQ decreased the level of DA (approximately $30 \%, P<0.01$ ) and that 
Fig. 3 The influence of acute (a) and chronic

(b) administration of 1MeTIQ on the elevation rate of dopamine catabolism induced by chronic treatment with 1BnTIQ. 1BnTIQ (25 mg/kg i.p.) was administered chronic during 14 consecutive days. 1MeTIQ ( 25 or $50 \mathrm{mg} / \mathrm{kg}$ i.p) was given acute (a) or chronic during 14 consecutive days (b). In the mixed group, 1MeTIQ was injected $20 \mathrm{~min}$ before 1BnTIQ administration. A control group was treated by saline. Rats were decapitated $2 \mathrm{~h}$ after the injections. The data are expressed as the

means \pm SEM $(n=6-7$ animals). Data were analyzed with a two-way ANOVA, followed by Duncan's post hoc test. Statistical significance:

* $P<0.05$; ** $P<0.01$ versus saline-treated group;

${ }^{++} P<0.01$ versus 1 BnTIQtreated group a

STRIATUM
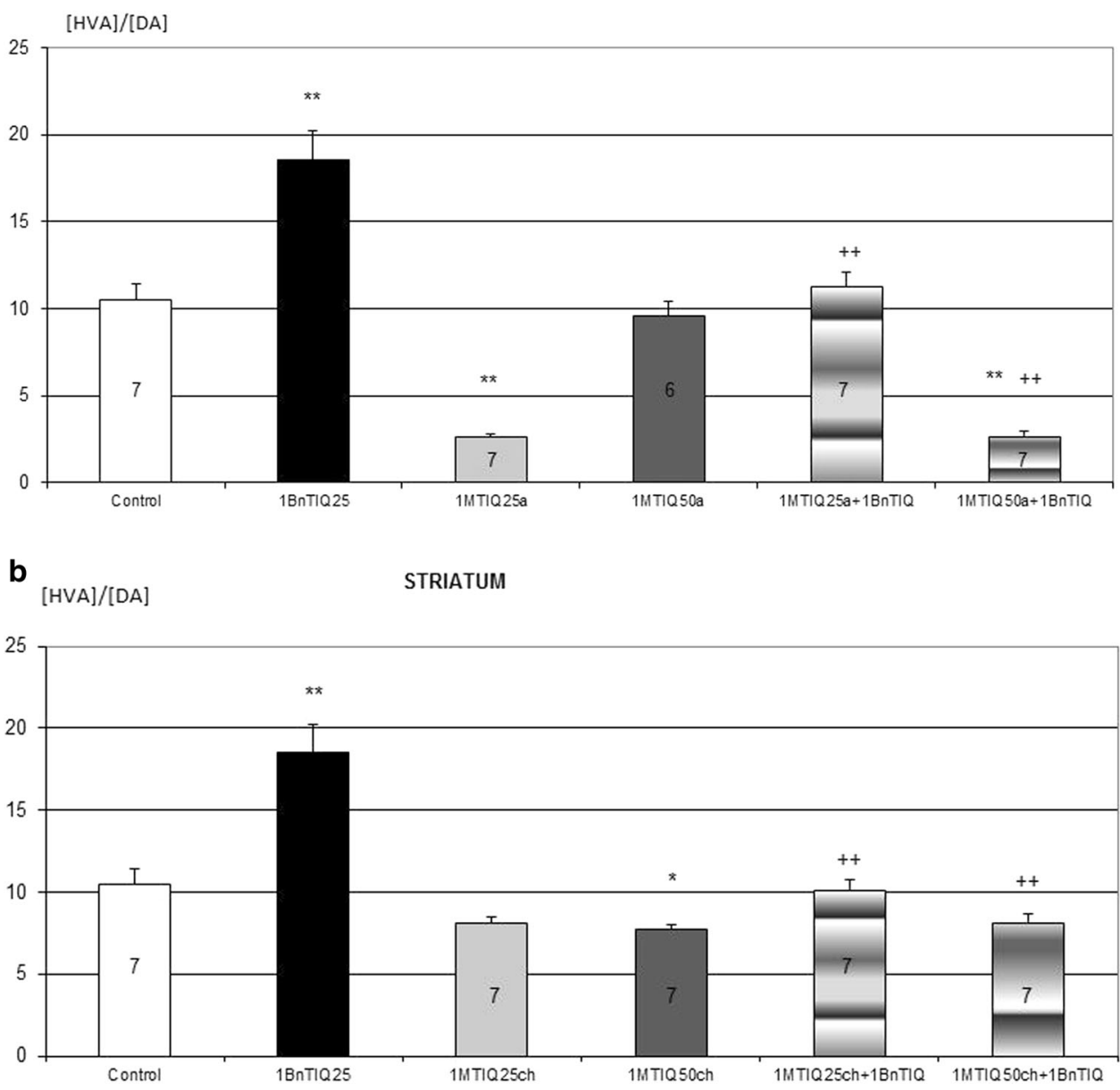

this effect was antagonized after chronic treatment with 1MeTIQ (Table 3).

Two-way ANOVA demonstrated a significant effect of chronic treatment with 1BnTIQ $(F[2,36]=29.92$, $P<0.01)$ and the multiple administration of $1 \mathrm{MeTIQ}$ $(F[2,36]=159.28, P<0.01)$ on the DOPAC concentration in rat nucleus accumbens (Table 3). An interaction between 1BnTIQ and L-DOPA was also detected $(F[2,36]=5.74, P<0.01)$. The post hoc analysis showed that chronic injections of both 1BnTIQ and 1MeTIQ reduced the DOPAC concentration (respectively, 25, 55 and $60 \% ; P<0.01)$; this effect was intensified in the mixed groups (respectively, 65 and $70 \%$ ) (Table 3).

Two-way ANOVA revealed no effect of the chronic administration of 1 BnTIQ $(F[2,36]=2.05$, N.S. $)$ on the level of 3-MT in the nucleus accumbens. The same statistical analysis indicated that chronic treatment with 1MeTIQ $(F[2,36]=14.17, P<0.01)$ significantly affected the 3-MT concentration (Table 3). The statistical analysis revealed no interaction between 1BnTIQ and 1MeTIQ $(F[2,36]=2.48$, N.S. $)$. Duncan's post hoc test showed that multiple treatment with 1BnTIQ decreased the level of 3-MT (approximately $30 \% ; P<0.05$ ), and this effect was reversed through chronic treatment at both concentrations of 1MeTIQ (Table 3).

Two-way ANOVA revealed a significant effect of chronic treatment with both 1BnTIQ $(F[2,36]=8.25$, $P<0.01)$ and 1MeTIQ $(F[2,36]=39.26, P<0.01)$ on the HVA concentration in the nucleus accumbens (Table 3). The interaction between the chronic administration of 1BnTIQ and chronic treatment with 1MeTIQ was not significant $(F[2,36]=0.33$, N.S. $)$. The post hoc analysis demonstrated that chronic treatment with both doses of 1MeTIQ strongly decreased the HVA concentration (respectively, 30 and $40 \% ; P<0.01)$; this effect was intensified in the mixed groups (approximately $50 \%$ ) (Table 3 ).

The Effect of Chronic 1MeTIQ Administration on $1 B n T I Q$ Produced Changes in the Concentration of EAA in the Rat Frontal Cortex (Table 4) One-way ANOVA demonstrated a significant effect of treatment $(F[6,35]=4.77$, $P<0.01)$ on the concentration of aspartate in the frontal cortex (Table 4). Duncan's post hoc test showed that the chronic administration of 1BnTIQ increased the aspartate 
Table 4 The influence of chronic treatment with $1 \mathrm{MeTIQ}$ and 1BnTIQ on the concentration of aspartate (ASP) and glutamate (GLU) in rat front al cortex

\begin{tabular}{llll}
\hline Treatment & $N$ & ASP $(\mathrm{ng} / 20 \mu \mathrm{l})$ & GLU $(\mathrm{ng} / 20 \mu \mathrm{l})$ \\
\hline $\begin{array}{l}\text { Frontal cortex } \\
\text { Saline }\end{array}$ & 5 & $464 \pm 15$ & \\
$1 \mathrm{BnTIQ} / 25 \times 1$ & 7 & $447 \pm 17$ & $1998 \pm 53$ \\
$1 \mathrm{BnTIQ} / 25 \times 14$ & 6 & $588 \pm 42^{* *}$ & $2005 \pm 42$ \\
$1 \mathrm{MeTIQ} / 25 \times 14$ & 5 & $498 \pm 10$ & $2273 \pm 161^{*}$ \\
$1 \mathrm{MeTIQ} / 50 \times 14$ & 6 & $520 \pm 21$ & $2133 \pm 100$ \\
$1 \mathrm{MeTIQ} / 25 \times 14+1 \mathrm{BnTIQ} / 25 \times 14$ & 6 & $477 \pm 16^{++}$ & $1988 \pm 38$ \\
$1 \mathrm{MeTIQ} / 50 \times 14+1 \mathrm{BnTIQ} / 25 \times 14$ & 6 & $530 \pm 15$ & $1998 \pm 55^{+}$ \\
$F$ & & $F_{(6 / 35)}=4.77$ & $2067 \pm 45$ \\
& & $P<0.01$ & $F_{(6 / 35)}=1.65$ \\
\hline
\end{tabular}

1BnTIQ (25 mg/kg i.p.) was administered acute or chronically during 14 consecutive days. 1MeTIQ was given in two doses (25 and $50 \mathrm{mg} / \mathrm{kg}$ i.p.) chronically. In the mixed group, 1MeTIQ was given also chronically, $20 \mathrm{~min}$ before each 1BnTIQ administration. Rats were decapitated $2 \mathrm{~h}$ after the injections. The results are expressed as the means \pm SEM of five to seven samples ( $n=5-7$ animals per group). Data were analyzed with a one-way ANOVA followed by Duncan's post hoc test. Statistical significance: ${ }^{*} P<0.05$; ** $P<0.01$ versus saline group; ${ }^{+} P<0.05 ;{ }^{++} P<0.01$ versus 1 BnTIQ group concentration (approximately $125 \% ; P<0.01$ ) and that this effect was completely inhibited through the chronic administration of 1MeTIQ $(P<0.01)$. The statistical analysis revealed no effect of treatment $(F[6,35]=1.65$, N.S.) on the level of glutamate in the frontal cortex (Table 4). However, post hoc analysis indicated that the chronic administration of 1BnTIQ increased the concentration of glutamate $(P<0.05)$ and that this effect was blocked through the multiple administration of 1MeTIQ (25 mg/kg i.p.) (Table 4).

\section{Immunoblotting}

Figure 4 shows that the acute administration of 1BnTIQ (25 $\mathrm{mg} / \mathrm{kg}$ i.p.) did not change the level of $\alpha$-synuclein in the substantia nigra, whereas the multiple administration of 1BnTIQ weakly (not significant) elevated the $\alpha$-synuclein concentration. A similar effect (non-significant increase in the level of $\alpha$-synuclein) was observed after the chronic administration of $1 \mathrm{MeTIQ}(50 \mathrm{mg} / \mathrm{kg}$ i.p.) and in the combined group (Fig. 4).

As demonstrated in Fig. 5, neither acute nor chronic treatment with 1 BnTIQ ( $25 \mathrm{mg} / \mathrm{kg}$ i.p.) changed the concentration of tyrosine hydroxylase. In addition, treatment with $1 \mathrm{MeTIQ}$ (50 mg/kg i.p.) alone and concomitant with 1BnTIQ did not influence the level of tyrosine hydroxylase (Fig. 5).

\section{Discussion}

The chronic administration of 1BnTIQ induced parkinsonism in primates, and this effect might reflect idiopathic Parkinson's disease (PD) (Abe et al. 2005). The neurotoxin
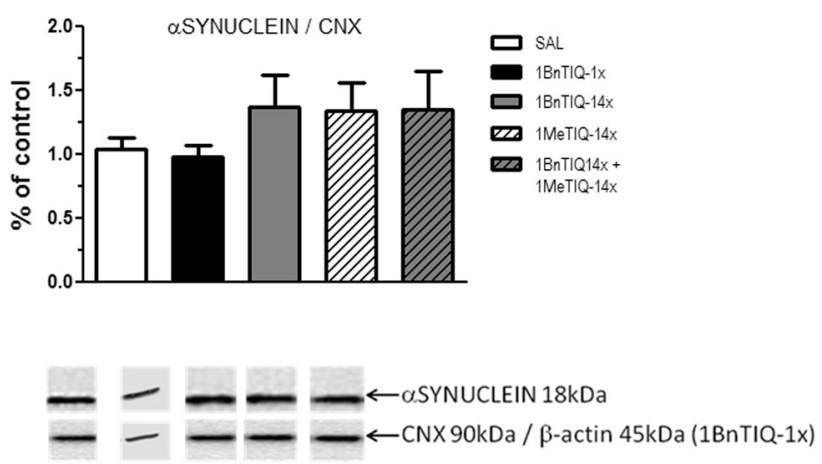

Fig. 4 The influence of chronic 1MeTIQ administration on 1BnTIQinduced changes in $\alpha$-synuclein level. 1BnTIQ was administered (25 $\mathrm{mg} / \mathrm{kg}$ i.p.) acute or chronically for 14 consecutive days. In the mixed group, $1 \mathrm{MeTIQ}(50 \mathrm{mg} / \mathrm{kg}$ i.p.) was administered chronically, $20 \mathrm{~min}$ before 1BnTIQ administration. Control rats were treated with the appropriate vehicle. Rats were killed by decapitation $2 \mathrm{~h}$ after last drug injections and substantia nigra was dissected for analysis. The data are expressed as the means \pm SEM. ( $n=6-7$ animals). Data were analyzed with a one-way ANOVA, followed by Duncan's post hoc test. Statistical significance: $* P<0.05$; $* * P<0.01$ versus saline-treated group; ${ }^{+} P<0.05$ versus 1 BnTIQ-treated group

1BnTIQ causes parkinsonism in humans, and this molecule is present in the cerebrospinal fluid of parkinsonian patients at approximately three times the concentration detected in healthy individuals (Kotake et al. 1995). These data clearly demonstrated that chronic treatment with a low dose of 1BnTIQ could represent a good animal model of PD. Distinct disturbances in the function of dopamine neurons generated through the repeated administration of 1BnTIQ might imitate the progressive character of PD. The results obtained in the present study confirm those of earlier studies and show that both the acute and chronic administration of 1BnTIQ significantly disturbed dopamine (DA) 

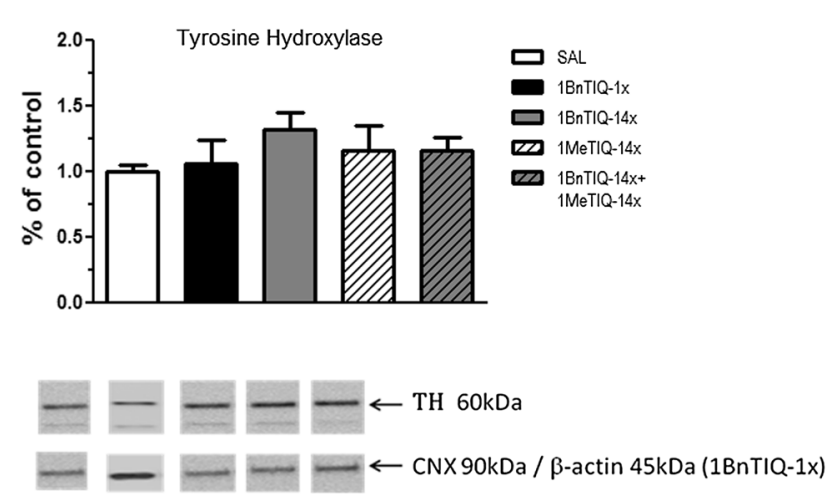

Fig. 5 The influence of chronic 1MeTIQ administration on 1BnTIQinduced changes in tyrosine hydroxylase concentration. 1BnTIQ was administered ( $25 \mathrm{mg} / \mathrm{kg}$ i.p.) acute or chronically for 14 consecutive days. In the mixed group, 1MeTIQ (50 mg/kg i.p.) was administered chronically, $20 \mathrm{~min}$ before 1BnTIQ administration. Control rats were treated with the appropriate vehicle. Rats were killed by decapitation $2 \mathrm{~h}$ after last drug injections and substantia nigra was dissected for analysis. The data are expressed as the means \pm SEM. $(n=6-7$ animals). Data were analyzed with a one-way ANOVA, followed by Duncan's post- hoc test. Statistical significance: $* P<0.05$; ** $P<0.01$ versus saline-treated group; ${ }^{+} P<0.05$ versus $1 \mathrm{BnTIQ}-$ treated group

metabolism in the rat brain (Wąsik et al. 2009). Interestingly, acute treatment with 1BnTIQ produced a stronger effect on DA metabolism than multiple administration, which suggests the development of tolerance after the chronic administration of 1BnTIQ (Table 1). However, both the single and repeated administration of 1BnTIQ significantly reduced the DA concentration in the brain structures, associated with the DA-mediated oxidative stress, as observed in the surviving dopaminergic terminals of PD patients (Antkiewicz-Michaluk et al. 2001). Previous studies have shown that 1BnTIQ strongly potentiates MAO-dependent DA oxidation, thereby increasing free radical production and damage to DA cells (Wassik et al. 2009). However, 1BnTIQ inhibits COMT activity and significantly decreases the concentration of 3-MT, an extraneuronal metabolite of DA, with a different neuroprotective effect (Miller et al. 1996). We also present the first investigation of the influence of the chronic administration of 1BnTIQ on the level of the excitatory amino acids (EAA), aspartate (ASP) and glutamate (GLU), in rat frontal cortex.

Using this animal model of PD, we investigated the effect of the neuroprotective endogenous amine, 1MeTIQ. As an endogenous inhibitor of MAO activity, 1MeTIQ shifts DA catabolism toward COMT-dependent $O$-methylation, thereby increasing the concentration of the extraneuronal DA metabolite, 3-MT (Antkiewicz-Michaluk et al. 2001; Patsenka and Antkiewicz-Michaluk 2004). Indeed, we previously observed that 1MeTIQ possesses neuroprotective activity in different animal models of neurodegeneration and protects neurons against glutamateand kainite-induced excitotoxicity (Antkiewicz-Michaluk et al. 2006, 2014).

In the present study, we demonstrated that both the acute and repeated administration of 1MeTIQ completely reversed the toxic effects of 1 BnTIQ on DA neurons. Additionally, the chronic administration of 1MeTIQ antagonized the 1BnTIQ-induced increase of the EAA concentration in the rat frontal cortex (Table 4). These data suggest that 1MeTIQ might participate in the EAA transmission; however, in the present study, we investigated the entire pool of GLU localized in the tissue. The chronic administration of 1BnTIQ increased the total level of EAA, potentially leading to excitotoxicity. Psychostimulants, such as nicotine, also increase the concentration of GLU in the prefrontal cortex, which increases neuronal activity (Falasca et al. 2014; Shameem and Patel 2012). The data from other studies have suggested that the systemic administration of the well-known neurotoxin 1-methyl-1phenyl-1,2,3,6-tetrahydropyridine (MPTP) in monkeys increases glutamatergic neurotransmission as an essential step in the evolution of parkinsonian symptoms (Bergman et al. 1990; Mitchell et al. 1989). The glutamate antagonist kynurenate reverses akinesia in MPTP-treated marmosets following focal injection into the medial pallidum (Brotchie et al. 1991). These results indicate that the selective reduction of glutamatergic overactivity might be an effective strategy for the treatment of PD.

The behavioral test showed that chronic treatment with 1BnTIQ significantly elevated the locomotor activity in rats (Fig. 1). Interestingly, an acute dose of 1BnTIQ (50 $\mathrm{mg} / \mathrm{kg}$ i.p.) produced the opposite effect, showing the reduction of exploratory activity in rats, as previously demonstrated (Wąsik et al. 2009). The hyperactivity of rats observed after the multiple administration of 1BnTIQ might reflect the 1BnTIQ-induced impairment of DA storage, and this reserpine-like inhibition of the function of vesicular monoamine transporter (VMAT) has been previously demonstrated in an in vivo microdialysis study (Wassik et al. 2014). Importantly, both the acute and chronic administration of 1MeTIQ (50 mg/kg i.p.) completely antagonized 1BnTIQ-induced hyperactivity (Fig. 1a, b) and reduced exploratory locomotor activity below that in the control group. Notably, the development of tolerance after the repeated administration of 1MeTIQ was not observed. This effect of 1MeTIQ can be explained by two mechanisms: (1) As a partial dopamine agonist, 1MeTIQ could block the active conformation of the DA receptor (Antkiewicz-Michaluk et al. 2014); and (2) 1MeTIQ shifts DA catabolism toward COMT-dependent $O$-methylation pathway, thereby significantly increasing the extraneuronal metabolite concentration of 3-MT (Antkiewicz-Michaluk et al. 2001). Moreover, 3-MT shows 
affinity to the $\alpha$-adrenergic receptor as an antagonist, and this molecule might play an important physiological role as an inhibitory regulator, counteracting the excessive stimulation of catecholaminergic neurons (Antkiewicz-Michaluk et al. 2008).

Biochemical studies have shown that the acute and chronic administration of 1BnTIQ markedly decreased the dopamine level in the extrapyramidal (striatum) and mesolimbic (nucleus accumbens) dopamine structures in the rat brain (Table 1). Post-mortem clinical trials demonstrated a total degradation of nigrostriatal pathway, but also other dopaminergic and noradrenergic structures (Tong et al. 2006).The toxic effect of 1 BnTIQ was completely blocked through the acute and chronic administration of 1MeTIQ (25 and $50 \mathrm{mg} / \mathrm{kg}$ ) in both investigated structures: striatum and nucleus accumbens (Tables 2, 3). The simultaneous administration of 1MeTIQ with 1BnTIQ not only restored the DA concentration to the control level but also decreased the DOPAC concentration, thereby inhibiting DA oxidation (Tables 2,3). Antagonism of 1MeTIQ compared to changes induced by 1 BnTIQ is better marked in the striatum (Tables 2, 3). We also demonstrated (Figs. 2, 3) that 1BnTIQ significantly elevated both the rate of the final DA catabolism, calculated as the ratio $[\mathrm{HVA}] /[\mathrm{DA}]$, and DA oxidation, calculated as the index [DOPAC]/[DA]. Both the acute and chronic administration of 1MeTIQ completely antagonized the effect of 1BnTIQ on the rate of DA catabolism (Figs. 2, 3). The biochemical effects of 1MeTIQ might reflect the restrictive influence of this molecule on MAO activity. It has been previously demonstrated that 1MeTIQ acts as a reversible inhibitor of MAO-A and MAOB activity (Patsenka and Antkiewicz-Michaluk 2004; Patsenka and Michaluk 2004; Antkiewicz-Michaluk et al. 2006). Interesting is that L-deprenyl (which similarly to 1MeTIQ is an MAO inhibitor) decreases the endogenous 1BnTIQ content in the mouse brain (Kotake et al. 1998).

Additionally, we showed that the chronic administration of 1BnTIQ increased the concentration of both ASP and GLU in the rat frontal cortex (Table 4). In this case, the chronic administration of 1MeTIQ at a lower dose (25 mg/ $\mathrm{kg}$ ) antagonized this effect. These results are consistent with previous in vitro experiments, demonstrating the inhibition through 1MeTIQ glutamate-induced excitotoxicity, particularly that mediated through NMDA receptors, and this effect might be an important factor in the mechanism of neuroprotection through 1MeTIQ (AntkiewiczMichaluk et al. 2006).

The potential neuroprotective activity of 1MeTIQ is of obvious interest because this compound is present in mammalian brains (Yamakawa and Ohta 1997, 1999; Yamakawa et al. 1999). These data are consistent with in vitro studies reporting that 1 MeTIQ prevents the neurotoxic action of the four dopaminergic neurotoxins,
MPTP, 6-OHDA, rotenone, and 1BnTIQ, in cultured mesencephalic neurons (Kotake et al. 2005). However, in the present study, acute and chronic treatment with 1BnTIQ did not change the levels of alpha-synuclein protein in the substantia nigra (Fig. 4). Moreover, the data obtained in the present study showed no effect of the repeated administration of 1BnTIQ on the tyrosine hydroxylase $(\mathrm{TH})$ concentration in the substantia nigra (Fig. 5). Similar effects were obtained by Abe et al. (2001a), they reported that administration of 1BnTIQ did not reduce TH-positive cells in the substantia nigra in C57BL mouse. Notably, in vitro studies presented different results from in vivo studies. Shavali et al. (2004) demonstrated that 1BnTIQ increased $\alpha$-synuclein expression and caused nuclear damage in human dopaminergic cells. Indeed, we should consider that in vivo experiments are not always consistent with in vitro research using cell culture. In vivo experiments often demonstrate compensatory mechanisms that might mask the toxic effects of the substance used. However, the other authors used TH immunohistochemistry to establish in vivo a potential protective effect of 1MeTIQ in the case when the death of dopaminergic cells was induced by MPP+ and TIQ (Abe et al. 2001b; Parrado et al. 2000). These papers confirmed the neuroprotective activity of $1 \mathrm{MeTIQ}$, and demonstrated a significant antagonism of 1MeTIQ against neurotoxininduced loss of TH-positive cells in the substantia nigra.

In conclusion, the results obtained in the present study clearly confirm that 1 MeTIQ possesses essential neuroprotective properties in an animal model of Parkinson's disease. Additionally, the neuroprotective effect of 1MeTIQ did not induce the development of tolerance after chronic administration and might restore the function of dopamine neurons, even after partial damage, which suggests their clinical relevance.

Acknowledgments The authors would like to thank Maria Kafel and Krzysztof Michalski for technical assistance. This study was financially supported through a Grant from the Polish Committee of Scientific Research, Grant No. DEC-2012/07/B/NZ7/01149, and statutory funds from the Institute of Pharmacology, Polish Academy of Sciences, Krakow, Poland.

Open Access This article is distributed under the terms of the Creative Commons Attribution 4.0 International License (http://creative commons.org/licenses/by/4.0/), which permits unrestricted use, distribution, and reproduction in any medium, provided you give appropriate credit to the original author(s) and the source, provide a link to the Creative Commons license, and indicate if changes were made.

\section{References}

Abe K, Taguchi K, Wasai T, Ren J, Utsunomiya I, Shinohara T, Miyatake T, Sano T (2001a) Biochemical and pathological study of endogenous 1-benzyl-1,2,3,4-tetrahydroisoquinoline-induced parkinsonism in the mouse. Brain Res 907:134-138 
Abe K, Taguchi K, Wasai T, Ren J, Utsunomiya I, Shinohara T, Miyatake T, Sano T (2001b) Stereoselective effect of (R)- and (S)-1-methyl-1,2,3,4-tetrahydroisoquinolines on a mouse model of Parkinson's disease. Brain Res Bull 56:55-60

Abe K, Saitoh T, Horiguchi Y, Utsunomiya I, Taguchi K (2005) Synthesis and neurotoxicity of tetrahydroisoquinoline derivatives for studying Parkinson's disease. Biol Pharm Bull 28:1355-1362

Antkiewicz-Michaluk L, Michaluk J, Mokrosz M, Romańska I, Lorenc-Koci E, Otha S, Vetulani J (2001) Different action on dopamine catabolic pathways of two endogenous 1,2,3,4tetrahydroisoquinolines with similar antidopaminergic properties. J Neurochem 78:100-108

Antkiewicz-Michaluk L, Łazarewicz JW, Patsenka A, Kajta M, Ziemińska E, Salińska E, Wąsik A, Gołembiowska K, Vetulani J (2006) The mechanism of 1,2,3,4-tetrahydroisoquinolines neuroprotection: the importance of free radicals scavenging properties and inhibition of glutamate-induced excitotoxicity. J Neurochem 97:846-856

Antkiewicz-Michaluk L, Filip M, Michaluk J, Romańska I, Przegaliński E, Vetulani J (2007) An endogenous neuroprotectant substance, 1-methyl-1,2,3,4-tetrahydroisoquinoline (1MeTIQ), prevents the behavioral and neurochemical effects of cocaine reinstatement in drug-dependent rats. J Neural Transm 114:307-317

Antkiewicz-Michaluk L, Ossowska K, Romańska I, Michaluk J, Vetulani J (2008) 3-Methoxytyramine, an extraneuronal dopamine metabolite plays a physiological role in the brain as an inhibitory regulator of catecholaminergic activity. Eur J Pharmacol 599:32-35

Antkiewicz-Michaluk L, Wąsik A, Michaluk J (2014) 1-Methyl1,2,3,4-tetrahydroisoquinoline, an endogenous amine with unexpected mechanism of action: new vistas of therapeutic application. Neurotox Res 25:1-12

Bergman H, Wichmann T, DeLong MR (1990) Reversal of experimental Parkinsonism by lesions of the subthalamic nucleus. Science 249:1436-1438

Brotchie JM, Mitchell IJ, Sambrook MA, Crossman A (1991) Alleviation of Parkinsonism by antagonism of excitatory amino acid transmission in the medial segment of globus pallidus in rat and primate. Mov Disord 6:133-138

Cannon JG, Webster GL (1958) Polyphosphoric acid in the BischlerNapieralski reaction. J Am Pharm Assoc Am Pharm Assoc 47(5):353-355

Falasca S, Ranc V, Petruzziello F, Khani A, Kretz R, Zhang X, Rainer $\mathrm{G}$ (2014) Altered neurochemical levels in the rat brain following chronic nicotine treatment. J Chem Neuroanat 59-60:29-35

Kohta R, Kotake Y, Hosoya T, Hiramatsu T, Otsubo Y, Koyama H, Hirokane Y, Yokoyama Y, Ikeshoji H, Oofusa K, Suzuki M, Ohta S (2010) 1-Benzyl-1,2,3,4-tetrahydroisoquinoline binds with tubulin beta, a substrate of parkin, and reduces its polyubiquitination. J Neurochem 114:1291-1301

Kotake Y, Tasaki Y, Makino Y, Otha S, Hirobe M (1995) 1-Benzyl1,2,3,4-tetrahydroisoquinoline as a parkinsonism-inducing agent: a novel endogenous amine in mouse brain and parkinsonian CSF. J Neurochem 65(6):2633-2638

Kotake Y, Tasaki Y, Hirobe M, Ohta S (1998) Deprenyl decreases an endogenous parkinsonism-inducing compound, 1-benzyl1,2,3,4-tetrahydroisoquinoline in mice: in vivo and in vitro studies. Brain Res 787:341-343

Kotake Y, Ohta S, Kanazawa I, Sakurai M (2003) Neurotoxicity of an endogenous brain amine, 1-benzyl-1,2,3,4-tetrahydroisoquinoline, in organotypic slice co-culture of mesencephalon and striatum. Neuroscience 117:63-70

Kotake Y, Taguchi R, Okuda K, Sekiya Y, Tasaki Y, Hirobe M, Ohta S (2005) Neuroprotective effect of 1-methyl-1,2,3,4- tetrahydroisoquinoline on cultured rat mesencephalic neurons in the presence or absence of various neurotoxins. Brain Res 1033(2):143-150

Miller J, Selhub J, Joseph J (1996) Oxidative damage caused by free radicals produced during catecholamine autooxidation: protective effects of O-methylation and melatonin. Free Radic Biol Med 21:241-249

Mitchell IJ, Clarke CE, Boyce S, Robertson RG, Peggs D, Sambrook MA, Crossman AR (1989) Neural mechanisms underlying parkinsonian symptoms based upon regional uptake of 2-deoxyglucose in monkeys exposed to 1-methyl-4-phenyl1,2,3,6-tetrahydropyridine. Neuroscience 32:213-226

Parrado J, Absi E, Ayala A, Castano A, Cano J, Machado A (2000) The endogenous amine 1-methyl-1,2,3,4-tetrahydroisoquinoline prevents the inhibition of complex I of the respiratory chain produced by $\mathrm{MPP}^{+}$. J Neurochem 75:65-71

Patsenka A, Antkiewicz-Michaluk L (2004) Inhibition of rodent brain monoamine oxidase and tyrosine hydroxylase by endogenous compounds-1,2,3,4-tetrahydro-isoquinoline alkaloids. Pol J Pharmacol 56:727-734

Patsenka A, Michaluk J, Antkiewicz-Michaluk L (2004) 1,2,3,4Tetrahydroisoquinoline alkaloids as endogenous inhibitors of brain monoamine oxidase, tyrosine hydroxylase and uptake of monoamines: in vitro study. In 13th international symposium on molecular and physiological aspects of regulatory processes of the organism. Kraków, Poland, Materials 2004, p 344

Shameem M, Patel AB (2012) Glutamatergic and GABAergic metabolism in mouse brain under chronic nicotine exposure: implications for addiction. PLoS ONE 7:e41824

Shavali S, Ebadi M (2003) 1-Benzyl-1,2,3,4-tetrahydroisoquinoline (1BnTIQ), an endogenous neurotoxin, induces dopaminergic cell death through apoptosis. Neurotoxicology 24:417-424

Shavali S, Carlson EC, Swinscoe JC, Ebadi M (2004) 1-Benzyl1,2,3,4-tetrahydroisoquinoline, a Parkinsonism-inducing endogenous toxin, increases $\alpha$-synuclein expression and causes nuclear damage in human dopaminergic cells. J Neurosci Res 76:563-572

Tong J, Hornykiewicz O, Kish SJ (2006) Inverse relationship between brain noradrenaline level and dopamine loss in Parkinson disease: a possible neuroprotective role for noradrenaline. Arch Neurol 63(12):1724-1728

Wąsik A, Romańska I, Antkiewicz-Michaluk L (2009) 1-Benzyl1,2,3,4-tetrahydroisoquinoline, an endogenous parkinsonism-inducing toxin, strongly potentiates MAO-dependent dopamine oxidation and impairs dopamine release: ex vivo and in vivo neurochemical studies. Neurotox Res 15:23-31

Wąsik A, Romańska I, Michaluk J, Kajta M, Antkiewicz-Michaluk L (2014) 1-Benzyl-1,2,3,4-tetrahydroisoquinoline, an endogenous neurotoxic compound, disturbs the behavioral and biochemical effects of 1-DOPA: in vivo and ex vivo studies in the rat. Neurotox Res 26(3):240-254

Yamakawa T, Ohta S (1997) Isolation of 1-methyl-1,2,3,4-tetrahydroisoquinoline-synthesizing enzyme from rat brain: a possible Parkinson's disease-preventing enzyme. Biochem Biophys Res Commun 236:676-681

Yamakawa T, Ohta S (1999) Biosynthesis of a parkinsonismpreventing substance, 1-methyl-1,2,3,4-tetrahydroisoquinoline, is inhibited by parkinsonism-inducing compounds in rat brain mitochondrial fraction. Neurosci Lett 259:157-160

Yamakawa T, Kotake Y, Fujitani M, Shintani H, Makino Y, Ohta S (1999) Regional distribution of parkinsonism-preventing endogenous tetrahydroisoquinoline derivatives and an endogenous parkinsonism-preventing substance-synthesizing enzyme in monkey brain. Neurosci Lett 276:68-70 